\title{
Quantitative Compression Optical Coherence Elastography as an Inverse Elasticity Problem
}

\author{
Li Dong, Philip Wijesinghe, James T. Dantuono, David D. Sampson, \\ Peter R.T. Munro, Brendan F. Kennedy, and Assad A. Oberai
}

March 9, 2016

\begin{abstract}
Quantitative elasticity imaging seeks to retrieve spatial maps of elastic moduli of tissue. Unlike strain, which is commonly imaged in compression elastography, elastic moduli are intrinsic properties of tissue and so this approach reconstructs images that are largely operator and system independent, enabling objective, longitudinal and multi-site diagnoses. Recently, novel quantitative elasticity imaging approaches to compression elastography have been developed. These methods use a calibration layer with known mechanical properties to sense the stress at the tissue surface, which combined with strain, is used to estimate the tissue's elastic moduli by assuming homogeneity in the stress field. However, this assumption is violated in mechanically heterogeneous samples. We present a more general approach to quantitative elasticity imaging that overcomes this limitation through an efficient iterative solution of the inverse elasticity problem using adjoint elasticity equations. We present solutions for linear elastic, isotropic and incompressible solids; however, this method can be employed for more complex mechanical models. We retrieve the spatial
\end{abstract}

${ }^{*}$ L. Dong, J. T. Dantuono and A. A. Oberai are with the Scientific Computation Research Center (SCOREC), Rensselaer Polytechnic Institute, Troy, NY 12180, USA (email: dongl3@rpi.edu; dantuj@rpi.edu; oberaa@rpi.edu)

${ }^{\dagger}$ P. Wijesinghe and B. F. Kennedy are with the Optical+Biomedical Engineering Laboratory, School of Electrical, Electronic and Computer Engineering, The University of Western Australia, Crawley, WA 6009, Australia (e-mail: philip.wijesinghe@research.uwa.edu.au; brendan.kennedy@uwa.edu.au)

${ }^{\ddagger}$ D. D. Sampson is with the Optical+Biomedical Engineering Laboratory, School of Electrical, Electronic and Computer Engineering, The University of Western Australia, Crawley, WA 6009, Australia and also with the Centre for Microscopy, Characterisation and Analysis, The University of Western Australia, Crawley, WA 6009, Australia (e-mail: david.sampson@uwa.edu.au).

${ }^{\S}$ P. R. T. Munro is with the Department of Medical Physics and Biomedical Engineering, University College London, WC1E 6BT, UK and the School of Electrical, Electronic and Computer Engineering, The University of Western Australia, Crawley, WA 6009, Australia (e-mail: p.munro@ucl.ac.uk) 
distribution of shear modulus for a tissue simulating phantom and a tissue sample. This is the first time, to our knowledge, that the iterative solution of the inverse elasticity problem has been implemented on experimentally acquired compression optical coherence tomography data.

\section{Introduction}

It has been well established that the mechanical properties of soft tissues are inextricably linked to the form and function of disease pathologies [1-4]. For instance, fibrosis in liver has been observed to increase its stiffness through increased production and decreased degradation of extracellular matrix constituents [5]. During metastasis, invasive cancer cells have been reported to reduce the stiffness of the surrounding extracellular matrix, aiding in their own migration through healthy tissues [6,7], whilst developed tumors, such as ductal carcinoma and fibroadenoma of the breast, have been characterized to be up to 14 times stiffer than surrounding fatty tissue $[8,9]$.

Knowledge of the mechanical properties can provide fundamental insight into the state of disease $[2,6,10]$; indeed, the biomechanics approach to identifying and characterizing disease has been emerging in the recent literature [3]. Particularly, a family of imaging techniques, termed elastography, have been developed to spatially map the mechanical properties of tissue into images, termed elastograms, on different length scales [11-13]. Ultrasound (US) and magnetic resonance (MR) elastography have been developed for whole organ imaging, with resolutions of $100 \mu \mathrm{m}$ and $1 \mathrm{~mm}$ [14], respectively, reaching commercial success by demonstrating significant improvement in disease diagnosis within a clinically relevant environment, primarily in characterizing breast cancer $[15,16]$ and liver fibrosis [17]. Over the past 15 years, optical coherence elastography (OCE), based on optical coherence tomography (OCT), has been following the precedent set by US and MR; however, on a finer length scale of tissue, with resolution down to $10 \mu \mathrm{m}[18,19]$. OCE has demonstrated potential in a wide range of applications, including breast cancer [20,21], ophthalmology [22, 23], and cardiology [24-26].

US, MR and optical elastography, at their core, involve the measurement of local displacement in tissue, which is elicited by some form of mechanical stimulation. From the displacement, it is possible to obtain some measure parameterizing tissue's mechanical behavior, such as local strain [27, 28], phase velocity $[29,30]$, creep $[31,32]$ or resonant vibration amplitude $[24,33]$. Such measurements, however, depend intrinsically upon the experimental method and tissue geometry, and do not usually represent a one-to-one mapping of the tissue's mechanical properties. Quantitative elastography uses some form of mathematical inversion, which we will describe in this paper, to retrieve quantitative elasticity images, i.e., quantitative maps of a sam- 
ple's intrinsic mechanical properties, which should be largely independent from the tissue loading, geometry and boundary conditions .

This process of mathematical inversion entails solving an inverse problem. The solution of such inverse problems is the subject of a large body of work, which we briefly introduce here before considering it in more detail in Section 2.1. There are broadly two principal ways in which the inverse elasticity problem can be solved: direct and iterative. The direct approach can be employed using so-called first-order approximations such as simplified models of tissue, limiting the accuracy of recovered elastic moduli [18]. When such first-order approximations are not made, direct approachs perform poorly when the measured data is incomplete or noisy [34]. Iterative approaches have been studied extensively in US and MR elastography [34]; however, few have implemented them for OCE $[35,36]$. Iterative methods allow for the inverse elasticity problem to be solved within a more general framework, requiring fewer assumptions, at the cost of higher computational complexity.

In this paper, we apply an iterative approach, using an adjoint method [37] to solve the inverse elasticity problem in compression OCE, reconstructing the shear modulus in a tissue-simulating phantom and airway tissue. Furthermore, the retrieved spatial distributions of shear modulus are made absolute by the use of a calibration layer of known stiffness. This is the first time that an iterative solution of the inverse elasticity problem has been applied in OCE using experimental, rather than synthetic $[35,36]$ data. The iterative approach makes fewer assumptions about the nature of mechanical deformation than direct approaches, which will likely lead to an improvement in the accuracy of reconstructed elastic moduli, and may enable more accurate longitudinal, multi-site and inter-sample comparison.

\section{Background}

\subsection{Quantitative elasticity imaging}

Quantitative elasticity imaging refers to the measurement, or estimation, of local intrinsic mechanical properties of a sample. The extracted properties must be premised on a mathematical model assumed to describe the underlying mechanical interactions under study. The most general model of tissue mechanics would include, for example, viscoelasticity, poroelasticity, anisotropy, and a nonlinear relationship between stress and strain [38,39]. It is usually necessary, and indeed reasonable, to neglect several, application specific properties in order to quantify one, or perhaps two, of the most significant tissue mechanical properties, the most common of which being the elastic moduli.

Quantitative elasticity imaging should disentangle, as far as is possible, the elastic properties of the sample from its aggregate mechanical behavior, 
which is obfuscated by the loading method, sample geometry and boundary conditions. If performed correctly, it means that quantitative elasticity images from different samples, acquired at different sites using different imaging systems, should be able to be compared quantitatively. Such techniques allow for the development of objective methods of assessing or diagnosing disease. They also support longitudinal studies by allowing measurements, taken over the course of a study, to be compared. Finally, quantitative elasticity imaging also reduces, or eliminates, artifacts introduced by instrument operators.

There is a substantial body of prior work on the subject of quantitative elasticity imaging, spanning imaging modalities such as US, MR and optical. Within each modality, a variety of physical models are employed to link applied load and measured displacement to elasticity parameters. Furthermore, within a particular modality and mechanical model, a variety of methods exist for solving the inverse elasticity problem, i.e., the retrieval of elasticity parameters from the measured displacement. Good reviews of this subject were published recently by Barbone and Oberai [13] and Doyley [34], to which we direct the reader for a comprehensive treatment of this subject. We summarise here the state of the art in order to provide the context for the current work.

\section{$2.2 \quad$ Assumed model of tissue mechanical properties}

Quantitative elasticity imaging methods are differentiated by the particular model of tissue mechanics upon which they are based. Assuming, as we do in this case, incompressible, linear elastic, isotropic tissue, one starts with the most general model, composed of the time varying equation of equilibrium and constitutive relation. This assumption means that instead of having potentially 21 independent elasticity parameters, we are left with only one [34], with Young's modulus, $E$, being related to shear modulus, $\mu$, as $E=3 \mu$. The principal models of tissue mechanics then differ according to how the equation of equilibrium's temporal dependence is treated. The three prevailing approaches are: quasi-static (as employed in this paper), harmonic and transient, in accordance with the principal ways in which all partial differential equations with a temporal dependence can be analysed. Equations (2)-(4), upon which our method is based, can be derived from the aforementioned general equations by assuming a quasi-static loading.

\subsection{First-order solutions to the inverse elasticity problem}

One approach to performing quantitative elasticity imaging is to implement simplified mechanical models which are readily invertible, either algebraically or numerically. These methods are often termed as first-order approximations [34] as they allow for measured displacements to be linearly 
related to elastic moduli. These models simplify the estimation of elastic moduli but with the penalty of using a potentially unrealistic model, which limits the accuracy of estimated elastic moduli in mechanically heterogeneous tissues possessing complex boundary conditions. A first-order approach to quasi-static elastography has been employed by researchers using US [40], MR [41] and OCT [42]. Under this approximation, the stress throughout a sample is assumed to be approximately constant. The strain throughout the sample, estimated from the measured displacement field, may, thus, be used directly in combination with Hooke's law to obtain Young's modulus. This approach works well in small tissue samples possessing only weak variations in Young's modulus [43], yet will likely break down in general, heterogeneous cases.

First-order approximations have also been applied to harmonic elastography in US [44], MR [45] and OCT [46]. One way in which a first-order modulus retrieval can be performed is to note that plane-wavefront shear waves propagate in homogeneous media with a velocity given by [1]:

$$
c_{s}=\sqrt{\frac{\mu}{\rho}},
$$

where $\mu$ is the shear modulus and $\rho$ is the mass density. Thus, under the assumption of plane shear propagation, which applies in a homogeneous region, shear modulus can be accurately extracted from measured values of shear wave velocity. This approach clearly breaks down in the presence of mechanical inhomogeneities and requires a priori knowledge of tissue density. Whilst not all harmonic techniques are based on this shear wave relationship, in general, they all rely on some approximation based on homogeneous wave propagation or resonance, which limits their generality.

Transient first-order models have also been applied in US [47] and MR [48] elastography. These methods were introduced to overcome the limited depth penetration of shear waves. Transient elastography uses an ultrasound transducer to remotely generate shear waves within tissue. This approach to quantitative elasticity imaging is considered a first-order approximation as it also uses (1) to relate shear wave velocity to shear modulus, and in so doing assumes that the shear wave travels in a reflection-free medium. Although schemes have been proposed to overcome this limitation [34], using such approaches for quantitative elasticity imaging in heterogeneous tissue still appears challenging.

First-order methods have also been applied in the field of optical elastography, including in optical coherence elastography (OCE). Such a method has recently been applied in quasi-static OCE by assuming that stress remains constant throughout a sample [42]. It has been used in a transient mode, where surface acoustic waves are generated using an air-puff [49] and a pulsed laser $[50,51]$. The group velocity of the resulting surface waves was measured using OCT $[49,51]$. In another approach, low-coherence interfer- 
ometry was used to measure the surface wave dispersion relationship [50]. In the former two cases [49,51], Young's modulus is retrieved from the surface wave group velocity, assuming that the surface wave travels on the boundary of an infinite half-space. In the latter case [50], a depth-resolved estimate of Young's modulus is obtained by analysing the dispersion relationship of the surface wave's spectral components, by exploiting the fact that lower frequency surface waves penetrate deeper into tissue. First-order harmonic approaches which make use of surface wave phase velocity to make depth-dependent estimates of Young's modulus have also been demonstrated $[29,52]$. The analytic relationship expressed in (1) has also been exploited within the context of harmonic optical coherence elastography to estimate Young's modulus within tissue from the phase velocity of shear waves [53]. Finally, we note that magnetomotively actuated optical coherence elastography can also be considered a harmonic first-order quantitative technique [54], [55]. In one example [55], tissue samples were assumed to be in the form of cylinders fixed to a base but otherwise free. An analytic model was then used to predict resonant modes of longitudinal vibration of the cylinders based upon the physical (including Young's modulus) properties of the sample. This allows a sample's Young's modulus to be extracted so long as there is sufficient information about the sample's length, radius, density and Poisson's ratio.

First-order methods have limited generality as a result of the approximations required to obtain a readily invertible relationship between measured displacement and retrieved elasticity modulus. For example, common amongst the previously discussed approaches, are approximations such as assuming mechanical homogeneity or constant stress. A different approach to solving the inverse elasticity problem is required if these, and other, assumptions are not to be made. Beyond first-order approaches, and for each temporal flavor of elastography as stated, there are two principal ways of solving the inverse elasticity problem: direct and iterative.

\subsection{Direct, general solutions of the inverse elasticity problem}

Direct methods of solving the elasticity inverse problem begin with a mathematical statement of the direct elasticity problem and transform this into a problem that may be solved directly for the tissue mechanical properties. One example of a direct approach using quasi-static elastography derives a set of coupled partial differential equations, which may be solved to yield the shear modulus and hydrostatic pressure within tissue [56]. Although direct approaches have been proposed for harmonic and transient approaches to elastography, we will focus on quasi-static methods since this is the focus of this paper, and the limitations of direct methods are common to all methods.

Although considerable work has been done on the use of direct inversion 
methods [56-59], they are not dominant, in general as a result of some weaknesses. The primary weakness is that these methods require accurate measurements of all components of the displacement field within the entire imaging plane. For example, in US elastography, accurate displacement measurements in any direction perpendicular to the direction of propagation of sound, are unfeasible. This limits the application of these methods.

\subsection{Iterative, general solutions of the elasticity inverse prob- lem}

An alternative to direct solution of the inverse elasticity problem is iterative solution. This approach allows solutions to be obtained which rely on, potentially, very few assumptions and approximations. Prior information, such as the expected range of values of shear modulus, can be integrated into the solution method. There is a considerable body of work on iterative solution methods to inverse problems, which provides tools that can be applied to the particular application of quantitative elasticity imaging [60]. Each iterative solution method is based upon a robust and realistic forward model, i.e., one which predicts displacements based upon given mechanical properties and applied load. The solution method iterates through candidate distributions of mechanical properties, ceasing when a satisfactory match has been reached between predicted and measured displacements as determined by an objective function such as (5), below. The main way in which iterative methods differ from one another is by how the estimate for mechanical properties is refined at each iteration.

Finally, we note that iterative solutions to the OCE inverse problem have previously been proposed $[35,36]$. In the first paper [36] to appear, the method of Kallel and Bertrand [61], the approximated Hessian iterative method, was employed. The paper presents results employing only synthetic data. The second paper to appear [35] differs significantly from the first, most likely due to the high computational overhead of requiring to calculate the Jacobian at each iteration. In this second work, the sample is segmented into a small number of (say up to four) regions with each region having a constant Young's modulus. The second difference is that the inverse problem is solved using a genetic algorithm, which would be a very computationally demanding approach for general problems such as that considered in this paper.

A classical approach to iteratively finding a solution which minimizes a functional such as (5) is to calculate the gradient vector and the Hessian matrix of (5) with respect to the spatially resolved elastic moduli, the unknowns of the inverse problem. Naïve approaches to calculating these quantities result in an inversion method that is computationally demanding. More advanced implementations [36, 61, 62] approximate the Hessian and evaluate the gradient in a significantly more efficient manner, which 
nonetheless increases in cost with the number of unknowns possessed by the inverse problem. An alternative method, which calculates the Jacobian of (5) by the solution of two direct problems, is known as the adjoint method [37], which results in a significant reduction in computational complexity, thus, making three-dimensional inverse problems tractable. It is this method which is employed in this paper.

\section{Methods}

\subsection{Computational method}

Optical coherence elastography, as considered here, yields spatially resolved measurements of tissue displacement which result from the quasi-static compression loading of tissue. Displacement fields are obtained using phasesensitive OCT [72] and are typically measured on a plane or within a volume, which we denote $\Omega$ in both cases. We express the measured displacement, mathematically, at location $\boldsymbol{x} \in \Omega$ as $\tilde{\boldsymbol{u}}(\boldsymbol{x})$. We note, however, that in general, only displacement components parallel to the OCT system's optical axis are measured, meaning that only one component of $\tilde{\boldsymbol{u}}(\boldsymbol{x}), \tilde{u}_{x_{3}}(\boldsymbol{x})$, is actually measured.

The time scale of the applied deformation is chosen such that the tissue inertia does not play a role in its mechanical response. As a result, the equations of equilibrium reduce to

$$
\nabla \cdot \boldsymbol{\sigma}=\mathbf{0}, \quad \text { in } \Omega,
$$

where $\boldsymbol{\sigma}$ is the Cauchy stress tensor and $\Omega$ represents the domain of the material. We model the material as an incompressible, isotropic solid, and assume a linear relation between stress and strain, which is reasonable since the applied strains are small. Under these assumptions, the stress-strain relation is given by

$$
\boldsymbol{\sigma}=-p \boldsymbol{I}+2 \mu \boldsymbol{\epsilon}
$$

where $p$ is the pressure, $\boldsymbol{I}$ is the identity tensor, $\boldsymbol{\epsilon}=\frac{1}{2}\left(\nabla \boldsymbol{u}+\nabla \boldsymbol{u}^{T}\right)$ is the strain tensor, $\boldsymbol{u}$ is the displacement vector, and $\mu$ is the shear modulus. The constraint of incompressibility implies that

$$
\operatorname{trace}(\boldsymbol{\epsilon})=0 .
$$

The forward elasticity problem may be stated as: given the shear modulus distribution in the domain $\Omega$, and displacement/traction boundary conditions on the boundary $\partial \Omega$, determine the displacement field and the pressure field, such that (2) and (4) are satisfied.

In the corresponding inverse problem, the displacement is known (measured), and the elastic parameter is sought. In particular, the inverse problem may be stated as: given the displacement field in $\Omega$, and displacement/traction boundary conditions on the boundary $\partial \Omega$, determine the 
shear modulus such that (2) and (4) are satisfied. Note that the pressure field is neither measured, nor is it explicitly sought. The pressure can be determined rather simply from (3) once the shear modulus is known.

When displacement data is available on a plane (as opposed to in a volume), a modeling choice has to be made. When the sample is relatively free to strain in the out-of-plane direction, it is conjectured that the stress components in this direction are small, and a state of plane-stress is assumed. On the other hand, when the sample is confined in the out-of-plane direction (due to boundary conditions, for example), it is conjectured that the strain in this direction is small, and a state of plane-strain is assumed. In this paper, due to boundary conditions at the top and bottom of the sample, the plane-strain assumption is made.

The solution to the inverse elasticity problem in three spatial dimensions, and in two dimensions in the plane strain configuration, is non-unique. In particular, there is an infinite set of solutions for $\mu$ that are consistent with the measured displacement field and equations (2) and (4). Prescribing traction data on the boundaries is equivalent to prescribing the shear modulus/pressure, and therefore, the existence of traction boundary conditions tends to reduce the solution set size of the inverse problem. For a detailed discussion of these topics, the reader is referred to [63-65].

The inverse problem is posed and solved as a constrained minimization problem. In particular, we seek the shear modulus distribution that minimizes the functional

$$
\pi=\frac{1}{2} \int_{\Omega}|\boldsymbol{T}(\boldsymbol{u}-\tilde{\boldsymbol{u}})|^{2} d \boldsymbol{x}+\alpha \int_{\Omega} \sqrt{|\nabla \mu|^{2}+c^{2}} d \boldsymbol{x},
$$

where the predicted displacement field, $\boldsymbol{u}$, is constrained to satisfy (2) and (4), and the prescribed boundary conditions. Further, $\boldsymbol{T}$ is a tensor whose values are used to weight displacement components according to their known or expected accuracy, $\alpha$ is a regularization parameter. $c$ is a numerical parameter $(c \leq|\nabla \mu|)$ that ensures the regularization term, which is a smoothed version of a total variation (TV) diminishing regularization [66], is a smooth function of $\mu$. In the examples considered in this manuscript, $c=0.01$. Further, it has been verified that in the regions where $\mu$ varies substantially, $c<<|\nabla \mu|$, and that the smoothed version of the TV term closely approximates the TV term. The first integral in (5) measures the mismatch between measured and predicted displacement, and is referred to as the displacement mismatch term. The second term is the regularization term added to account for the ill-posed nature of the inverse problem, i.e., small noise in the measured displacements leads to large, unphysical variations in the shear modulus distribution. These oscillations are suppressed by a large value of the regularization parameter which ensures smaller total variation in the recovered modulus distribution. It is worth noting that it does so at the expense of increasing the displacement matching term. 


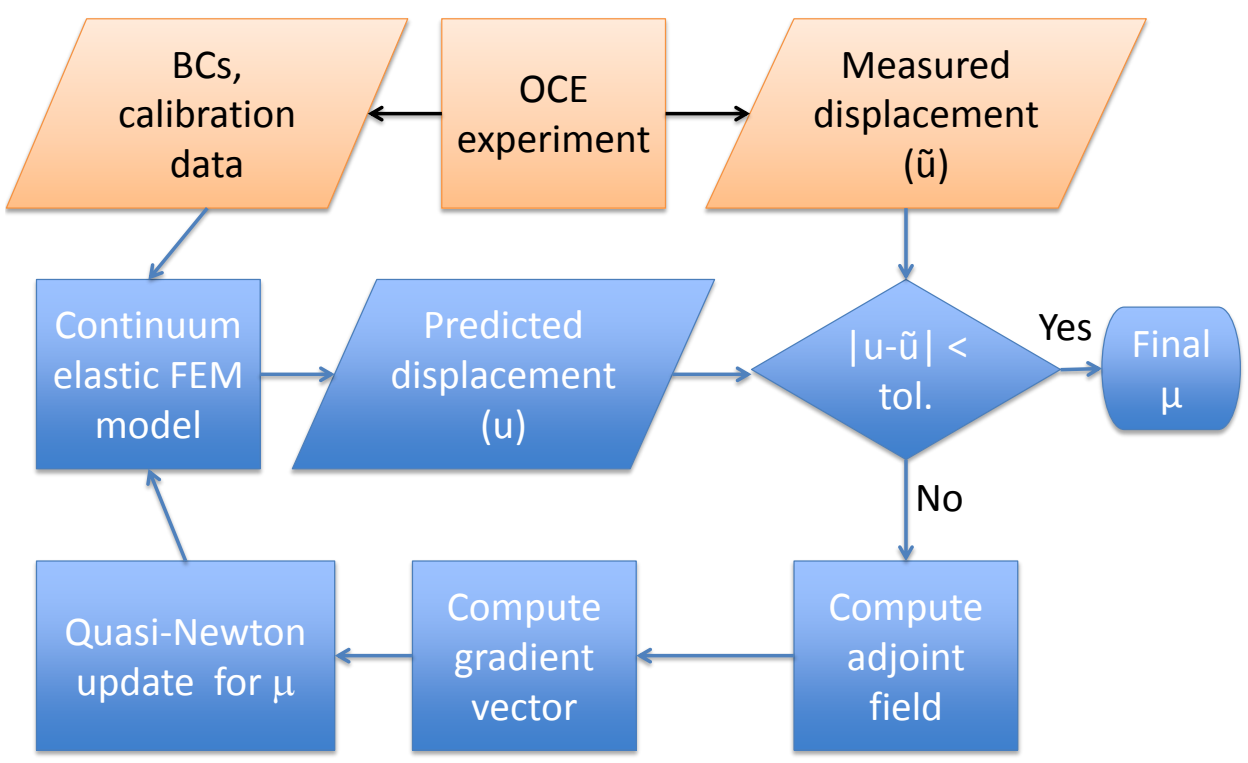

Figure 1: Flow diagram of the inverse problem solver (shaded blue) and how it interacts with the experimental system (shaded in orange). Computation concludes when the discrepancy between the predicted and measured displacement fields falls below a prescribed tolerance (denoted tol.).

The problem of minimizing $\pi$ reduces to a discrete optimization problem with nonlinear constraints once all the field variables are represented using finite element basis functions, and the constraint equations are discretized using the finite element method. The optimization parameters are the nodal values of the shear modulus field. This problem is solved using a quasiNewton method, as depicted in Fig. 1, that builds the Hessian information by repeatedly evaluating the gradient of $\pi$ with respect to the optimization parameters [67]. A straightforward evaluation of this gradient requires as many solves of the forward elasticity problem as the number of parameters, which can be very large $\left(O\left(10^{4}\right)\right.$ for our problems). A much more efficient approach is to derive and solve the adjoint equations for the adjoint field, and use the adjoint and the predicted displacement fields to evaluate the gradient vector $[37,68]$. Within this approach, the gradient of $\pi$ with respect to $\mu$ can be evaluated by solving a single forward and a single adjoint problem (which is of the same complexity as the forward problem) independent of the number of parameters used to represent the shear modulus. Considering that this number is $O\left(10^{4}\right)$, this is a significant, and necessary, computational saving. 


\subsection{Experimental setup}

A fiber-based Fourier-domain OCT system was employed to perform OCE. The imaging system is described in detail in [21], and briefly here. The system was set up in a common-path configuration, such that the reference signal is provided by the reflection from the interface of a 2-mm glass window and a sample situated upon it. A superluminescent diode source with a central wavelength of $835 \mathrm{~nm}$ and a bandwidth of $50 \mathrm{~nm}$ was used to illuminate the sample with $\sim 7.5 \mathrm{~mW}$ of power. The sensitivity was measured as $102 \mathrm{~dB}$ for an exposure time of $36 \mu \mathrm{s}$, and an exposure time of $2-3 \mu \mathrm{s}$ was used in general. The system provides axial and lateral resolutions of 6.1 and $11 \mu \mathrm{m}$, respectively, assuming a sample refractive index of 1.4 [69]. This refractive index was also used, throughout this paper, to scale the axial coordinates of the measured OCT intensities and displacements to closer match their true locations.

Compression OCE was performed by placing a sample on top of a 600$\mu \mathrm{m}$ thick compliant poly-dimethylsiloxane (PDMS) silicone layer of known stiffness, which was used to constrain the solution set of the inverse problem, hereby referred to as the calibration layer. Fabrication of the layer is described in detail in [69]. The sample-silicone assembly was situated between a rigid plate and a glass window coupled to a piezoelectric ring actuator [27], such that the OCT beam would pass though the window, then the silicone, and finally into the sample. To ensure even contact, the rigid plate was used to apply $5-10 \%$ preload strain. Following the preload, the piezoelectric actuator was used to apply step-wise micro-scale compression to the sample-silicone assembly, and two-dimensional images were captured sequentially, in the uncompressed and compressed (relative to the preload) states. The actuator stroke was set between 1 and $2 \mu \mathrm{m}$, to ensure a high displacement and strain sensitivity [71]. The maximum attainable displacement and strain sensitivity was measured previously as $0.34 \mathrm{~nm}$ and $2.6 \mu \varepsilon$ $\left(\mu \varepsilon\right.$ is microstrain, strain $\left.\times 10^{-6}\right)$, respectively [21]. Actuation frequency was set below $25 \mathrm{~Hz}$, which was experimentally verified to avoid any detectable wave propagation. We employ phase-sensitive OCE, described in detail in [72], in which the axial component of the displacement field, resulting from the micro-scale compression, is obtained from the difference between the angles of the polar-form complex OCT scans, corresponding to the uncompressed and compressed tissue states. Using this approach, we can measure axial displacement within the range $\pm 2.2 \mu \mathrm{m}[21]$.

\section{Results}

In this section, we demonstrate the solution of the inverse elasticity problem for two samples: a tissue-simulating silicone phantom and a transverse section of an equine bronchus. The silicone phantom incorporated a 830- $\mu \mathrm{m}$ 

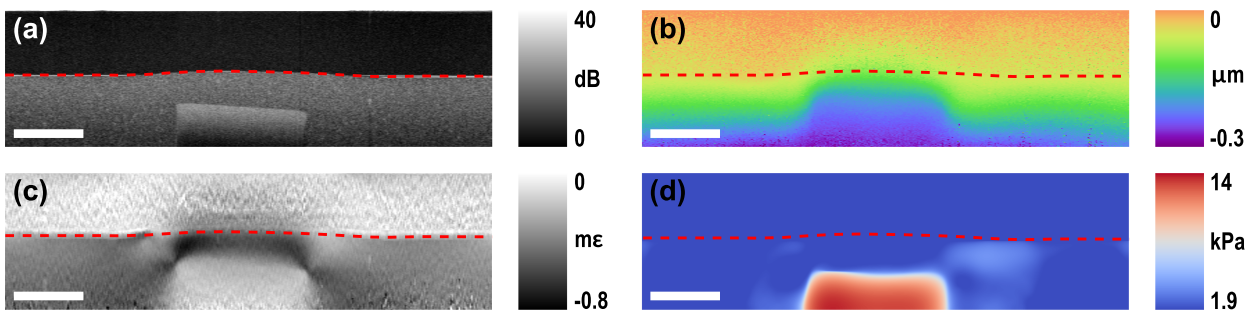

Figure 2: Tissue-simulating silicone phantom. (a) OCT image in $\mathrm{dB}$, (b) local displacement from micro-scale actuation, (c) axial strain, where $\mathrm{m} \varepsilon$ is milli-strain, and (d) shear modulus image, reconstructed by solving the elasticity inverse problem. The scale bar represents $500 \mu \mathrm{m}$. Red dashed lines represent the layer-tissue boundary.

wide, rectangular, stiff inclusion embedded approximately $300 \mu \mathrm{m}$ below the surface of a softer homogeneous matrix, following the fabrication method described in detail in [69]. The inclusion and the matrix were experimentally characterized using a standard bulk compression test (Instron, Norwood, MA, USA) to possess a shear modulus of 9.3 and $1.9 \mathrm{kPa}$, respectively, for preload applied in the experiment. The calibration layer, described in Section 3.2, was also characterized to have a shear modulus of $1.9 \mathrm{kPa}$.

The OCT image of the already preloaded phantom is presented in Fig. 2(a), in which we can identify the calibration layer (black) and the inclusion embedded in a matrix. The displacement due to the micro-scale compression provided by the piezoelectric actuator is presented in Fig. 2(b). It is apparent how the feature distorts the otherwise uniform displacement field gradient: the result of the contrast in stiffness metween it and the matrix. Fig. 2(c) shows an image of the axial strain, calculated as the gradient of axial displacement in depth [27], which constitutes a standard elastogram that would be produced by compression OCE [21]. Although we can identify the presence of the inclusion in the strain alone, it is evident that its relation to the stiffness of the material is distorted by the effects of material property heterogeneities, particularly apparent at the boundaries of the inclusion.

The axial component of the displacement data, Fig. 2(b), was used to infer the spatial distribution of the shear modulus using the method described in Section 3.2. This was achieved by setting $T_{x_{3} x_{3}}=1$, and all other components of $\boldsymbol{T}$ to zero. The shear modulus is presented in Fig. 2(d). Due to the relatively large out-of-plane extent of the phantom and the compression plate, a state of plane strain was assumed within the imaging plane. The OCT intensity map was used to determine the extent of the region within this plane over which the inverse problem was solved. Specifically, points with lower OCT intensity (and hence larger displacement noise) were discarded. This led to a rectangular domain of 982 by 3,500 microns (axial by lateral), containing 300 by 350 grid points; only this domain is presented in 

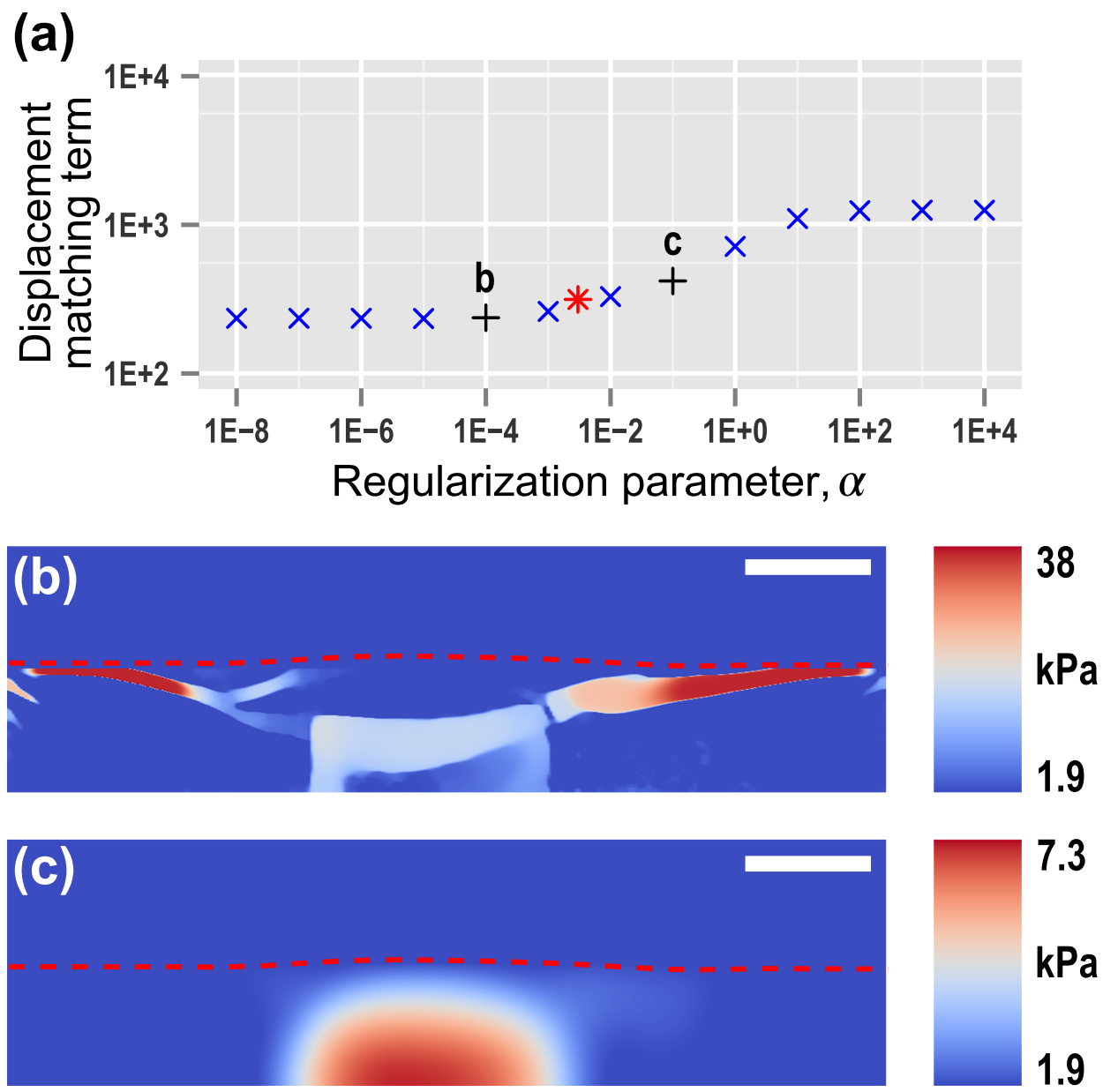

Figure 3: (a) Displacement matching term with respect to the regularization parameter in the inclusion phantom; red star marker shows the selected optimal regularization parameter used in Fig. 2(d). Shear modulus images evaluated with regularization parameters labeled by black plus markers are shown in (b) as under-regularized, and (c) over-regularized. Red dashed lines represent the layer-tissue boundary.

Fig. 2. On every boundary edge of this region the measured displacement was prescribed as a boundary condition along the axial direction. Along the lateral direction, zero traction was assumed on all edges except the edge in contact with the compression plate (the top edge), where, due to the stickiness of the contact, zero lateral displacement was enforced.

The value of the shear modulus in the calibration layer was held fixed at unity, and the shear modulus at every other point was determined by solving the inverse problem. This resulted in a problem with 52,150 optimization parameters. These parameters were constrained to lie in the interval $(1,20)$, 

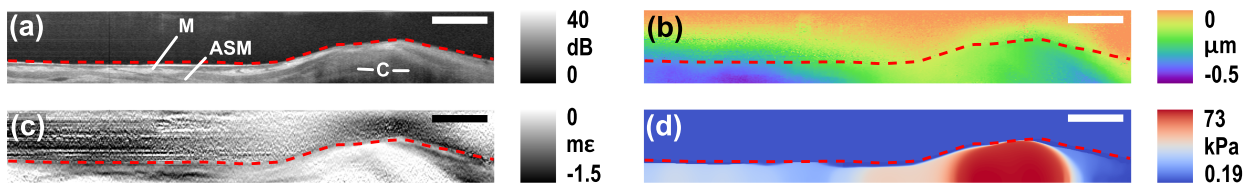

Figure 4: Transverse section of an equine bronchus. (a) OCT image in dB. M: mucosa, ASM: airway smooth muscle, and C: cartilage; (b) local displacement from micro-scale actuation; (c) axial strain, where $\mathrm{m} \varepsilon$ is milli-strain; and $(\mathrm{d})$ shear modulus image, reconstructed by solving the elasticity inverse problem. The scale bar represents $500 \mu \mathrm{m}$. Red dashed lines represent the layer-tissue boundary.
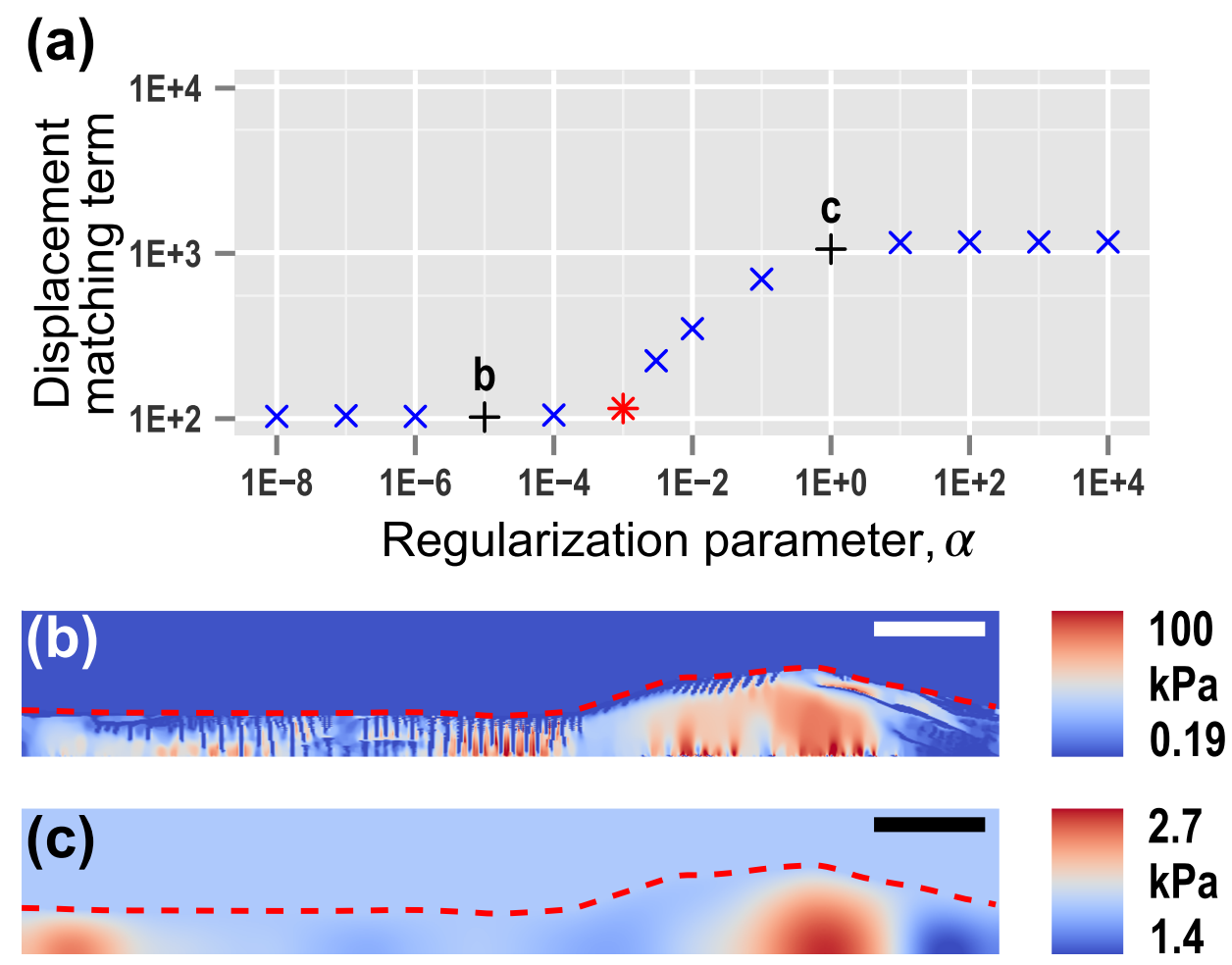

Figure 5: (a) Displacement matching term with respect to the regularization parameter in tissue; red star marker shows the selected optimal regularization parameter used in Fig. 4(d). Shear modulus images evaluated with regularization parameters labeled by black plus markers are shown in (b) as under-regularized, and (c) over-regularized. Red dashed lines represent the layer-tissue boundary.

and a uniform value of unity was used as an initial guess. The quasi-Newton algorithm used to solve the inverse problem was considered converged whenever the maximum number of iterations $(6,000)$ was achieved, or the change 
in the displacement matching term over the last 5 iterations was below a certain tolerance. We note that the modulus distribution in Fig. 2(d) is quantitative, and is obtained by multiplying the reconstructed modulus by the known value of the shear modulus $(1.9 \mathrm{kPa})$ in the calibration layer. We can see that by obtaining shear modulus, we can, to a significant extent, decouple the inclusion feature from strain artifacts. The average measured shear modulus in the inclusion and the matrix was measured to be 10.6 and $2.2 \mathrm{kPa}$, respectively, which was within $15 \%$ of the expected values. The upper and lower limits of the colormap in this and all modulus images in this paper were set equal to the minimum and maximum modulus values, respectively, within the domain.

A sweep was performed in order to determine the "optimal" value of the regularization parameter. For every value of the regularization parameter, an inverse problem was solved and the value of the displacement matching term was determined. This yielded a single data point on the curve plotted in Fig. 3(a). In these curves, we observe two asymptotes corresponding to small and large values of the regularization parameter. The largest value of the regularization parameter which yielded a displacement matching term close to the lower asymptote was selected to be the optimal value by using the L-curve [73]. This value $(\alpha=0.003)$ is indicated by the red marker in Fig. 3, and the corresponding modulus distribution is the one displayed in Fig. 2(d).

Maps of the reconstructed modulus at small and large values of the regularization parameter (values marked by the black markers in Fig. 3(a)) are shown in Fig. 3(b) and (c). These clearly demonstrate the smoothing effecton the modulus distribution of increasing the regularization term.

The OCT image of the transverse section of an equine bronchus is presented in Fig. 4(a). The lumenal side, i.e., the side facing the center of the bronchus, faces the top in the OCT image. Three clearly identifiable tissue types are labeled in Fig. 4(a), namely: the mucosa, airway smooth muscle and cartilage. The features were classified with the aid of histological sections, and previously classified OCT images of airway tissue [74]. Similarly to the phantom results, the displacement due to the micro-scale compression is presented in Fig. 4(b), and a standard strain elastogram in Fig. 4(c). We can see that both the mucosa and the airway smooth muscle exhibit higher compressive strain than the cartilage, consistent with the expected greater stiffness of the cartilage.

The axial component of the measured displacement data was used to infer the shear modulus presented in Fig. 4(d). Further, a state of plane strain was assumed, and the OCT image was used to determine the region of interest (ROI) for the inverse problem. A domain of size 524 by 4,400 microns (axial by lateral), with 160 by 440 grid points, was considered, and the same kind of boundary conditions, as for the tissue-phantom case, were imposed. Again, only this domain is presented in Fig. 4. 
The value of the shear modulus in the calibration layer was fixed at unity, which resulted in 26,379 optimization parameters, which were constrained to be in the interval $(0.1,100)$. Their initial value was set to unity, and the convergence criterion for the algorithm was unchanged from the tissuephantom problem. A sweep over the regularization parameter resulted in the curve plotted in Fig. 5(a). This curve was used to determine the optimal value of the regularization parameter, $\alpha=0.001$. The resulting shear modulus distribution, after rescaling by the modulus in the calibration layer $(1.9 \mathrm{kPa})$, is shown in Fig. 4(d). In this figure, we can clearly observe the cartilage as a very stiff region with a mean shear modulus of around $63 \mathrm{kPa}$. The modulus distributions at small and large values of the regularization parameter are shown in Fig. 5(b) and (c). Once again, they demonstrate the smoothing effect of the regularization term. In Fig. 5(b), we observe highly localized regions (just below the cartilage) where the modulus attains the upper bound $(190 \mathrm{kPa})$. This lack of smoothness is a consequence of a regularization parameter that is too small for the level of noise in the measured data.

\section{Discussion}

In this paper, we have formulated quantitative OCE as an inverse problem. We have solved this problem as a constrained minimization problem, wherein the objective function is a measure of the mismatch between the measured displacement field and a predicted displacement field, which is constrained to satisfy the equations of equilibrium in conjunction with a constitutive model. The nodal values of the material parameter(s) (in this paper, the shear modulus) of the constitutive model are the optimization parameters. A gradient-based optimization algorithm is used to drive the displacement matching term to its minima by computing iterative updates to these parameters. To our knowledge, this work presents the first instance of the application of the iterative solution of the inverse elasticity problem to quantitative OCE of tissue and tissue-mimicking phantoms. As discussed below, this approach has several advantages when compared with other quantitative OCE methods.

Iterative solution of the inverse elasticity problem is consistent with completely heterogeneous stress states, which are almost always found in any specimen with heterogeneous material properties. In contrast to this, most other OCE methods assume that the axial stress component is homogeneous along the axial direction. The violation of this assumption leads to artifacts in modulus images generated using these methods. These artifacts are absent in images constructed using our approach. This can be observed in the tissue-phantom study results by comparing the strain image in Fig. 2 (which is typically interpreted as the reciprocal of Young's modulus) to the 
reconstructed shear modulus image in the same figure. The former displays artifacts at the upper corners of the inclusion that are absent in the latter.

Another advantage of iteratively solving the elasticity inverse problem is its flexibility. For example, in this paper, we have assumed that the sample is in a state of plane-strain, because (a) the width of the sample along the out-of-plane direction is much larger than that of the imaging plane (field of view), and (b) the compression plate is in contact with most of the top surface of the sample. Due to these assumptions, the out-of-plane strain components are expected to be small. On the other hand, in a typical ultrasound elastography application, such as breast elastography, the tissue is unconfined and is compressed using the transducer itself. The footprint of the transducer then only covers a small fraction of the surface of the tissue. Consequently, there is significant strain in the direction normal to the Bscan imaging plane, and the situation is closer to that of plane-stress. The approach presented here allows for both plane-strain and plane-stress (and other) scenarios by simply modifying the constitutive relation (3) in the forward problem. The reader is referred to [75] for results obtained under the plane-stress hypothesis.

Our approach of iteratively solving the inverse elasticity problem also allows for flexibility in including prior information about measurement noise and material parameter distributions. For example, in this paper, we have independent estimates of the shear modulus of the calibration layer, and we know that its distribution is uniform. We make use of this knowledge to fix the modulus value in the calibration layer, and to rescale the reconstructed modulus in order to obtain quantitative modulus images. Also, the lateral component of displacement is unknown. We account for this by setting all but the axial component of the tensor $\boldsymbol{T}$ to zero. We note that in general this tensor should be set equal to the inverse of the covariance tensor for the noise in the displacement measurements.

The choice of the regularization term and the regularization parameter is an area of active research in inverse problems. We have used total variation (TV) regularization in this study because it penalizes variations in modulus distribution without smearing out sharp variations [66]. TV is appropriate for biomedical applications in which tissue types and/or pathologies are distinguished by sharp boundaries, which is often the case.

The regularization parameter can dramatically alter the reconstructed modulus results, with larger values leading to smoother distributions with very little contrast, and smaller values leading to sharp, noise-induced artifacts (see Figs. 2-5). An appropriate value of this parameter can be selected by using the L-curve (as was done in this paper) [73] or the Morozov discrepancy principle [76]. In the discrepancy principle, this parameter is selected so that the displacement mismatch term is approximately equal to the magnitude of the noise in the measured displacement. Both approaches are effective in determining an optimal parameter; however, both require mul- 
tiple solutions of the inverse problem, which is a computationally expensive problem to solve to begin with.

The robustness of the choice for the optimal value of the regularization parameter was tested by studying the effect of varying this value on the reconstructions (results not shown here). When the regularization parameter was increased by $50 \%$, the reconstructed contrast in the shear modulus for the tissue phantom decreased by about 5\%; whereas, the contrast in the equine bronchus specimen reduced by about $20 \%$. When the regularization parameter was decreased by $50 \%$, the shear modulus contrast in the tissue-phantom increased by $10 \%$, and the contrast in the equine bronchus specimen increased by about $20 \%$. These results demonstrate that our reconstructions are robust (to within 20\%) to the choice of the regularization parameter.

While the results presented in this paper validate the proposed approach and the choices made therein (plane-strain, boundary conditions, etc.), we believe that there is room for improvement in making these choices and for extensions of this approach. In particular, our plans for future work are as follows.

1. Solving the inverse elasticity problem on displacement data measured in a three-dimensional volume, to generate three-dimensional modulus images. In addition to the obvious advantage of generating volumetric modulus images, this would eliminate the plane-strain assumption and the inaccuracies that come with it. In fact, we have readily captured three-dimensional volumes of displacement previously [21] for qualitative strain imaging; however, the challenge in the reconstruction of three-dimensional modulus lies in addressing the computational expense of solving the inverse problem, which will invariably be increased from more optimization parameters introduced by the additional dimension.

2. Measuring lateral displacement components. Including lateral displacement data will make our reconstructions more robust and will provide data for boundary conditions along the lateral direction. Although phase-sensitive methods, used in this paper, provide access to only the axial component of displacement, other methods, such as speckle tracking $[77,78]$, have been used to measure all displacement components. Speckle tracking, natively, has a lower sensitivity to displacement than phase-sensitive methods: however, both can in principle be used in tandem, through incremental loading, to provide high-sensitivity axial, and low-sensitivity lateral displacement measures. The variance can be readily accounted for by generating a tensor $\boldsymbol{T}$, whose components are selected in inverse proportion to the magnitude of error in displacement measurements [27]. This accounts 
for the spatial variation of this error, as well as the differences in the error between the axial and lateral displacement components.

3. Applying additional loads which would lead to multiple independent displacement measurements. The inverse elasticity problem with just one displacement field is non-unique in three dimensions and in two dimensions under the plane-strain assumption. The uniqueness and the robustness of this inverse problem can be improved by measuring multiple, independent displacement fields $[64,65]$. One way to generate an independent field would be to compress the specimen after rotating the compression plate a little (say $10 \%$ ) about an axis that is perpendicular to the imaging plane.

4. Making incremental displacement measurements about significantly pre-stressed (about 10-30 \% strain) states. These displacement measurements will provide information about the nonlinear elastic response of the specimen, and in conjunction with a nonlinear hyperelastic constitutive model could be used to create maps of the nonlinear elastic parameters of tissue $[16,75]$.

5. Estimating the spatial resolution sensitivity of this approach to quantitative OCE. This is a challenging endeavor that is complicated by the fact that the resolution and sensitivity of the modulus image is determined by the resolution of the optical system, the algorithm used to estimate displacements, the spectrum of the inverse elasticity operator, the type of regularization employed in the inverse problem, the precise value of the regularization parameter, and all sources of noise.

\section{Conclusion}

We have demonstrated quantitative OCE: a method for performing quantitative elasticity imaging using compression OCE. This method is based upon the iterative solution of an inverse problem with the use of the adjoint equations to make the method computationally feasible. We applied the method to two examples: a tissue-mimicking phantom and an equine bronchus sample, both of which resulted in predicted distributions of shear modulus which were within the expected range. Although both examples were two-dimensional and within the plane-strain approximation, this method is amenable to solving three-dimensional problems. This method, is in fact, very general in that different types of prior information, boundary conditions, and tissue mechanical models can be incorporated into it. We anticipate that this approach to quantitative elasticity imaging will become an important tool in the study of biomechanics and medical imaging applications. 


\section{Acknowledgement}

LD and AAO are supported by the US NSF grant \#1148,111. PW is supported by the William and Marlene Schrader Scholarship provided by The University of Western Australia. PM is supported by a Royal Society University Research Fellowship and was supported by a Discovery Early Career Research Award from the Australian Research Council (DE120101331) during some of this work. This work is supported in part by the Australian Research Council Discovery scheme.

\section{References}

[1] K. J. Parker, L. S. Taylor, S. Gracewski, and D. J. Rubens, "A unified view of imaging the elastic properties of tissue," J. Acoust. Soc. Am., vol. 117, no. 5, p. 2705, 2005.

[2] D. Discher, C. Dong, J. J. Fredberg, F. Guilak, D. Ingber, P. Janmey, R. D. Kamm, G. W. Schmid-Schönbein, and S. Weinbaum, "Biomechanics: Cell research and applications for the next decade," Ann. Biomed. Eng., vol. 37, no. 5, pp. 847-859, May 2009.

[3] G. Y. Lee and C. T. Lim, "Biomechanics approaches to studying human diseases," Trends Biotechnol., vol. 25, no. 3, pp. 111-118, Mar. 2007.

[4] P. A. Janmey and C. A. McCulloch, "Cell mechanics: Integrating cell responses to mechanical stimuli," Annu. Rev. Biomed. Eng., vol. 9, no. 1, pp. 1-34, Aug. 2007.

[5] R. G. Wells, "The role of matrix stiffness in hepatic stellate cell activation and liver fibrosis," J. Clin. Gastroenterol., vol. 39, no. 4, pp. S158-S161, Apr 2005.

[6] P. Friedl and S. Alexander, "Cancer invasion and the microenvironment: plasticity and reciprocity," Cell, vol. 147, no. 5, pp. 992-1009, Nov. 2011.

[7] C. T. Mierke, "Endothelial cell's biomechanical properties are regulated by invasive cancer cells," Mol. Biosyst., vol. 8, no. 6, pp. 1639-1649, 2012 .

[8] P. Wellman, R. D. Howe, E. Dalton, and K. A. Kern, "Breast tissue stiffness in compression is correlated to histological diagnosis," Harvard BioRobotics Lab. Tech. Rep., 1999.

[9] T. Liu, O. A. Babaniyi, T. J. Hall, P. E. Barbone, and A. A. Oberai, "Noninvasive in-vivo quantification of mechanical heterogeneity of invasive breast carcinomas," PloS one, vol. 10, no. 7, p. e0130258, 2015. 
[10] D. Ingber, "Mechanobiology and diseases of mechanotransduction," Ann. Med., vol. 35, no. 8, pp. 564-577, 2003.

[11] K. J. Parker, M. M. Doyley, and D. J. Rubens, "Imaging the elastic properties of tissue: The 20 year perspective," Phys. Med. Biol., vol. 56, no. 1, pp. R1-R29, Jan. 2011.

[12] J. F. Greenleaf, M. Fatemi, and M. Insana, "Selected methods for imaging elastic properties of biological tissues," Annu. Rev. Biomed. Eng., vol. 5, no. 1, pp. 57-78, Aug. 2003.

[13] P. E. Barbone and A. A. Oberai, "A review of the mathematical and computational foundations of biomechanical imaging," in Computational Modeling in Biomechanics. Springer, 2010, pp. 375-408.

[14] J. Braun, J. Guo, R. Lützkendorf, J. Stadler, S. Papazoglou, S. Hirsch, I. Sack, and J. Bernarding, "High-resolution mechanical imaging of the human brain by three-dimensional multifrequency magnetic resonance elastography at 7T," Neuroimage, vol. 90, pp. 308-314, Apr. 2014.

[15] J. M. Chang, W. K. Moon, N. Cho, A. Yi, H. R. Koo, W. Han, D.-Y. Noh, H.-G. Moon, and S. J. Kim, "Clinical application of shear wave elastography (SWE) in the diagnosis of benign and malignant breast diseases," Breast Cancer Res. Treat., vol. 129, no. 1, pp. 89-97, Aug. 2011.

[16] S. Goenezen, J.-F. Dord, Z. Sink, P. E. Barbone, Jingfeng Jiang, T. J. Hall, and A. A. Oberai, "Linear and nonlinear elastic modulus imaging: An application to breast cancer diagnosis," IEEE Trans. Med. Imaging, vol. 31, no. 8, pp. 1628-1637, Aug. 2012.

[17] L. Sandrin, B. Fourquet, J. M. Hasquenoph, S. Yon, C. Fournier, F. Mal, C. Christidis, M. Ziol, B. Poulet, F. Kazemi, M. Beaugrand, and R. Palau, "Transient elastography: A new noninvasive method for assessment of hepatic fibrosis," Ultrasound Med. Biol., vol. 29, no. 12, pp. 1705-1713, 2003.

[18] B. F. Kennedy, K. M. Kennedy, and D. D. Sampson, "A review of optical coherence elastography: Fundamentals, techniques and prospects," IEEE J. Sel. Top. Quantum Electron., vol. 20, no. 2, pp. 272-288, Mar. 2014.

[19] S. Wang and K. V. Larin, "Optical coherence elastography for tissue characterization: A review," J. Biophotonics, vol. 8, no. 4, pp. 279-302, 2015. 
[20] B. F. Kennedy, R. A. McLaughlin, K. M. Kennedy, L. Chin, P. Wijesinghe, A. Curatolo, A. Tien, M. Ronald, B. Latham, C. M. Saunders, and D. D. Sampson, "Investigation of optical coherence microelastography as a method to visualize cancers in human breast tissue," Cancer Res., vol. 75, no. 16, pp. 3236-3245, Aug 2015.

[21] B. F. Kennedy, R. A. McLaughlin, K. M. Kennedy, L. Chin, A. Curatolo, A. Tien, B. Latham, C. M. Saunders, and D. D. Sampson, "Optical coherence micro-elastography: Mechanical-contrast imaging of tissue microstructure," Biomed. Opt. Express, vol. 5, no. 7, pp. 2113-2124, Jul. 2014.

[22] S. Wang and K. V. Larin, "Shear wave imaging optical coherence tomography (SWI-OCT) for ocular tissue biomechanics," Opt. Lett., vol. 39, no. 1, pp. 41-44, Jan. 2014.

[23] C. Li, G. Guan, Z. Huang, M. Johnstone, and R. K. Wang, "Noncontact all-optical measurement of corneal elasticity," Opt. Lett., vol. 37, no. 10, pp. 1625-1627, May 2012.

[24] W. Qi, R. Li, T. Ma, J. Li, K. Kirk Shung, Q. Zhou, and Z. Chen, "Resonant acoustic radiation force optical coherence elastography," Appl. Phys. Lett., vol. 103, no. 10, p. 103704, 2013.

[25] G. van Soest, F. Mastik, N. de Jong, and A. F. W. van der Steen, "Robust intravascular optical coherence elastography by line correlations," Phys. Med. Biol., vol. 52, no. 9, pp. 2445-2458, May 2007.

[26] A. H. Chau, R. C. Chan, M. Shishkov, B. MacNeill, N. Iftimiia, G. J. Tearney, R. D. Kamm, B. E. Bouma, and M. R. Kaazempur-Mofrad, "Mechanical analysis of atherosclerotic plaques based on optical coherence tomography," Ann. Biomed. Eng., vol. 32, no. 11, pp. 1494-1503, 2004 .

[27] B. F. Kennedy, S. H. Koh, R. A. McLaughlin, K. M. Kennedy, P. R. T. Munro, and D. D. Sampson, "Strain estimation in phase-sensitive optical coherence elastography," Biomed. Opt. Express, vol. 3, no. 8, pp. 1865-1879, Aug. 2012.

[28] A. Nahas, M. Bauer, S. Roux, and A. C. Boccara, "3D static elastography at the micrometer scale using full field OCT," Biomed. Opt. Express, vol. 4, no. 10, pp. 2138-2149, Oct 2013.

[29] C. Li, G. Guan, X. Cheng, Z. Huang, and R. K. Wang, "Quantitative elastography provided by surface acoustic waves measured by phasesensitive optical coherence tomography," Opt. Lett., vol. 37, no. 4, pp. 722-724, 2012. 
[30] R. K. Manapuram, S. Aglyamov, F. M. Menodiado, M. Mashiatulla, S. Wang, S. A. Baranov, J. Li, S. Emelianov, and K. V. Larin, "Estimation of shear wave velocity in gelatin phantoms utilizing PhS-SSOCT," Laser Phys., vol. 22, no. 9, pp. 1439-1444, Sep. 2012.

[31] P. Wijesinghe, R. A. McLaughlin, D. D. Sampson, and B. F. Kennedy, "Parametric imaging of viscoelasticity using optical coherence elastography," Phys. Med. Biol., vol. 60, no. 6, p. 2293, 2015.

[32] V. Crecea, A. Ahmad, and S. A. Boppart, "Magnetomotive optical coherence elastography for microrheology of biological tissues," J. Biomed. Opt., vol. 18, no. 12, p. 121504, 2013, 10.1117/1.JBO.18.12.121504.

[33] S. G. Adie, X. Liang, B. F. Kennedy, R. John, D. D. Sampson, and S. A. Boppart, "Spectroscopic optical coherence elastography," Opt. Express, vol. 18, no. 25, pp. 25 519-25 534, Dec 2010.

[34] M. M. Doyley, "Model-based elastography: A survey of approaches to the inverse elasticity problem," Phys. Med. Biol., vol. 57, no. 3, pp. R35-R73, Feb. 2012.

[35] A. S. Khalil, B. E. Bouma, and M. R. Kaazempur Mofrad, "A combined FEM/genetic algorithm for vascular soft tissue elasticity estimation," Cardiovasc. Eng., vol. 6, no. 3, pp. 93-102, 2006.

[36] A. S. Khalil, R. C. Chan, A. H. Chau, B. E. Bouma, and M. R. K. Mofrad, "Tissue elasticity estimation with optical coherence elastography: Toward mechanical characterization of in vivo soft tissue," Ann. Biomed. Eng., vol. 33, no. 11, pp. 1631-1639, Nov. 2005.

[37] A. A. Oberai, N. H. Gokhale, and G. Feijó, "Solution of inverse problems in elasticity imaging using the adjoint method," Inverse Probl., vol. 19, no. 2, pp. 297-313, Apr. 2003.

[38] F. A. Duck, Physical properties of tissue: A comprehensive reference book. London: Academic Press, 1990.

[39] Y. Fung, Biomechanics: Mechanical properties of living tissues, 2nd ed. New York: Springer-Verlag, 1993.

[40] J. Ophir, "Elastography: A quantitative method for imaging the elasticity of biological tissues," Ultrason. Imaging, vol. 13, no. 2, pp. 111-134, Apr. 1991.

[41] J. B. Fowlkes, "Magnetic-resonance imaging techniques for detection of elasticity variation," Med. Phys., vol. 22, no. 11, p. 1771, 1995. 
[42] K. M. Kennedy, L. Chin, R. A. McLaughlin, B. Latham, C. M. Saunders, D. D. Sampson, and B. F. Kennedy, "Quantitative microelastography: Imaging of tissue elasticity using compression optical coherence elastography," Sci. Rep., to be published.

[43] F. Kallel, J. Ophir, K. Magee, and T. Krouskop, "Elastographic imaging of low-contrast elastic modulus distributions in tissue," Ultrasound Med. Biol., vol. 24, no. 3, pp. 409-425, Mar. 1998.

[44] K. J. Parker, S. R. Huang, R. A. Musulin, ans R. M. Lerner, "Tissue response to mechanical vibrations for "sonoelastic imaging"," Ultrasound Med. Biol., vol. 16, no. 3, pp. 241-46, 1990.

[45] R. Muthupillai, D. Lomas, P. Rossman, J. Greenleaf, A. Manduca, and R. Ehman, "Magnetic resonance elastography by direct visualization of propagating acoustic strain waves," Science, vol. 269, no. 5232, pp. 1854-1857, Sep. 1995.

[46] A. L. Oldenburg, G. Wu, D. Spivak, F. Tsui, A. S. Wolberg, and T. H. Fischer, "Imaging and elastometry of blood clots using magnetomotive optical coherence tomography and labeled platelets," IEEE J. Sel. Top. Quantum Electron., vol. 18, no. 3, pp. 1100-1109, May 2012.

[47] A. P. Sarvazyan, O. V. Rudenko, S. D. Swanson, J. Fowlkes, and S. Y. Emelianov, "Shear wave elasticity imaging: A new ultrasonic technology of medical diagnostics," Ultrasound Med. Biol., vol. 24, no. 9, pp. 1419-1435, Dec. 1998.

[48] P. McCracken, A. Manduca, J. P. Felmlee, and R. L. Ehman, "Transient MR elastography: Modeling traumatic brain injury," Lancet, vol. 3217, pp. 1081-1082, 2004.

[49] S. Wang, J. Li, R. K. Manapuram, F. M. Menodiado, D. R. Ingram, M. D. Twa, A. J. Lazar, D. C. Lev, R. E. Pollock, and K. V. Larin, "Noncontact measurement of elasticity for the detection of soft-tissue tumors using phase-sensitive optical coherence tomography combined with a focused air-puff system," Opt. Lett., vol. 37, no. 24, pp. 51845186, Dec. 2012.

[50] C. Li, Z. Huang, and R. K. Wang, "Elastic properties of soft tissuemimicking phantoms assessed by combined use of laser ultrasonics and low coherence interferometry," Opt. Express, vol. 19, no. 11, pp. 1015363, May 2011.

[51] C. Li, G. Guan, Z. Huang, M. Johnstone, and R. K. Wang, "Noncontact all-optical measurement of corneal elasticity," Opt. Lett., vol. 37, no. 10, pp. 1625-1627, 2012. 
[52] K. D. Mohan and A. L. Oldenburg, "Elastography of soft materials and tissues by holographic imaging of surface acoustic waves," Opt. Express, vol. 20, no. 17, pp. 18887-97, Aug. 2012.

[53] M. Razani, A. Mariampillai, C. Sun, T. W. H. Luk, V. X. D. Yang, and M. C. Kolios, "Feasibility of optical coherence elastography measurements of shear wave propagation in homogeneous tissue equivalent phantoms," Biomed. Opt. Express, vol. 3, no. 5, pp. 972-980, 2012.

[54] V. Crecea, A. L. Oldenburg, X. Liang, T. S. Ralston, and S. A. Boppart, "Magnetomotive nanoparticle transducers for optical rheology of viscoelastic materials," Opt. Express, vol. 17, no. 25, pp. 23114-23122, 2009 .

[55] A. L. Oldenburg and S.A. Boppart, "Resonant acoustic spectroscopy of soft tissues using embedded magnetomotive nanotransducers and optical coherence tomography," Phys. Med. Biol., vol. 55, no. 4, pp. 1189-1201, 2010.

[56] K. Raghavan and A. Yagle, "Forward and inverse problems in elasticity imaging of soft tissues," IEEE Trans. Nucl. Sci., vol. 41, no. 4, pp. 1639-1648, 1994.

[57] C. Sumi, A. Suzuki, and K. Nakayama, "Phantom experiment on estimation of shear modulus distribution in soft tissue from ultrasonic measurement of displacement vector field," IEICE Trans. Fundam. Electron. Commun. Comput., vol. E78-A, no. 12, pp. 1655-1664, 1995.

[58] U. Albocher, A. A. Oberai, P. E. Barbone, and I. Harari, "Adjointweighted equation for inverse problems of incompressible plane-stress elasticity," Comput. Method. Appl. M., vol. 198, no. 30, pp. 2412-2420, 2009 .

[59] P. E. Barbone, C. E. Rivas, I. Harari, U. Albocher, A. A. Oberai, and Y. Zhang, "Adjoint-weighted variational formulation for the direct solution of inverse problems of general linear elasticity with full interior data," Int. J. Numer. Meth. Eng., vol. 81, no. 13, pp. 1713-1736, 2010.

[60] M. Burger, B. Kaltenbacher, and A. Neubauer, "Iterative solution methods," in Handb. Math. Methods Imaging. New York, NY: Springer New York, 2011, pp. 345-384.

[61] F. Kallel and M. Bertrand, "Tissue elasticity reconstruction using linear perturbation method," IEEE Trans. Med. Imaging, vol. 15, no. 3, pp. 299-313, Jun. 1996. 
[62] M. M. Doyley, P. M. Meaney, and J. C. Bamber, "Evaluation of an iterative reconstruction method for quantitative elastography," Phys. Med. Biol., vol. 45, no. 6, pp. 1521-1540, Jun. 2000.

[63] P. E. Barbone and J. C. Bamber, "Quantitative elasticity imaging: What can and cannot be inferred from strain images," Phys. Med. Biol., vol. 47, no. 12, pp. 2147-2164, Jun. 2002.

[64] P. E. Barbone and N. H. Gokhale, "Elastic modulus imaging: On the uniqueness and nonuniqueness of the elastography inverse problem in two dimensions," Inverse Probl., vol. 20, no. 1, pp. 283-296, Feb. 2004.

[65] U. Albocher, P. E. Barbone, A. A. Oberai, and I. Harari, "Uniqueness of inverse problems of isotropic incompressible three-dimensional elasticity," J. Mech. Phys. Solids, vol. 73, pp. 55-68, Dec. 2014.

[66] L. I. Rudin, S. Osher, and E. Fatemi, "Nonlinear total variation based noise removal algorithms," Phys. D Nonlinear Phenom., vol. 60, no. 1-4, pp. 259-268, Nov. 1992.

[67] C. Zhu, R. H. Byrd, P. Lu, and J. Nocedal, "Algorithm 778: L-BFGSB: Fortran subroutines for large-scale bound-constrained optimization," ACM Trans. Math. Softw., vol. 23, no. 4, pp. 550-560, Dec. 1997.

[68] N. H. Gokhale, P. E. Barbone, and A. A. Oberai, "Solution of the nonlinear elasticity imaging inverse problem: The compressible case," Inverse Probl., vol. 24, no. 4, p. 045010, Aug. 2008.

[69] G. Lamouche, B. F. Kennedy, K. M. Kennedy, C.-E. Bisaillon, A. Curatolo, G. Campbell, V. Pazos, and D. D. Sampson, "Review of tissue simulating phantoms with controllable optical, mechanical and structural properties for use in optical coherence tomography," Biomed. Opt. Express, vol. 3, no. 6, pp. 1381-1398, Jun. 2012.

[70] K. M. Kennedy, S. Es'haghian, L. Chin, R. A. McLaughlin, D. D. Sampson, and B. F. Kennedy, "Optical palpation: Optical coherence tomography-based tactile imaging using a compliant sensor," Opt. Lett., vol. 39, no. 10, pp. 3014-3017, May 2014.

[71] L. Chin, A. Curatolo, B. F. Kennedy, B. J. Doyle, P. R. T. Munro, R. A. McLaughlin, and D. D. Sampson, "Analysis of image formation in optical coherence elastography using a multiphysics approach," Biomedical Optics Express, vol. 5, no. 9, pp. 2913-2930, 2014.

[72] R. K. Wang, S. Kirkpatrick, and M. Hinds, "Phase-sensitive optical coherence elastography for mapping tissue microstrains in real time," Appl. Phys. Lett., vol. 90, no. 16, p. 164105, 2007. 
[73] A. Tarantola, Inverse problem theory and methods for model parameter estimation. Society for Industrial Mathematics, 2005.

[74] R. A. McLaughlin, P. B. Noble, and D. D. Sampson, "Optical coherence tomography in respiratory science and medicine: From airways to alveoli," Physiology, vol. 29, no. 5, pp. 369-380, Sep. 2014.

[75] A. A. Oberai, N. H. Gokhale, S. Goenezen, P. E. Barbone, T. J. Hall, A. M. Sommer, and J. Jiang, "Linear and nonlinear elasticity imaging of soft tissue in vivo: Demonstration of feasibility," Phys. Med. Biol., vol. 54, no. 5, pp. 1191-1207, Mar. 2009.

[76] V. A. Morozov, "On the solution of functional equations by the method of regularization," Sov. Math Dokl., vol. 7, pp. 414-417, 1966.

[77] J. Schmitt, "OCT elastography: Imaging microscopic deformation and strain of tissue." Opt. Express, vol. 3, no. 6, pp. 199-211, 1998.

[78] J. Fu, F. Pierron, and P. Ruiz, "Elastic stiffness characterization using three-dimensional full-field deformation obtained with optical coherence tomography and digital volume correlation," J. Biomed. Opt. Opt., vol. 18, no. 12, p. 121512, 2013. 